\title{
A Practical Subspace Multiple Measurement Vectors Algorithm for Cooperative Spectrum Sensing
}

\author{
Tsung-Hsun Chien ${ }^{1}$, Wei-Jie Liang ${ }^{1,2}$, and Chun-Shien $\mathrm{Lu}^{1}$ \\ ${ }^{1}$ Institute of Information Science, Academia Sinica, Taipei, Taiwan, ROC \\ ${ }^{2}$ Department of Mathematics, National Cheng-Kung University, Tainan, Taiwan, ROC
}

\begin{abstract}
Cooperative spectrum sensing (CSS) in cognitive radio networks conducts cooperation among sensing users to jointly sense the sparse spectrum and utilize available spectrums. Greedy multiple measurement vectors (MMVs) algorithm in the context of compressed sensing can ideally model the wideband CSS scenario to efficiently solve the support detection problem for identification of occupied channels. Actually, the number of sparsity is unknown, and most of greedy algorithms for MMVs lack for a (robust) stopping criterion of determining when the greedy algorithm should terminate. In this paper, we analyze and derive oracle stopping bounds for greedy MMVs algorithms without depending on prior information such as sparsity. Moreover, we introduce a practical subspace MMVs greedy algorithm that extends from a subspace-based sparse recovery method to a more practical setting, in which no prior information are required. Extensive simulations confirm the feasibility of the proposed stopping criteria and our sparse recovery algorithm.
\end{abstract}

Keywords-Compressed Sensing, (Cooperative) Spectrum Sensing, Matching pursuit, Multiple Measurement Vectors, Sparsity

\section{INTRODUCTION}

\section{A. Background}

Cognitive Radio [1] is one of solutions to efficiently solve sparse spectrum usage [2] in wireless communications. Secondary Users (SUs) are allowed to sufficiently exploit available spectrum, which are not currently used by Primary Users (PUs), via spectrum sensing (SS) techniques. Most existing methods are based on Nyquist sampling theorem for the purpose of exact recovery of the original signal. Therefore, they require large sampling rates under wideband spectrum sensing scenarios [3].

With an eye on the fact [2] that only few spectra will be used, i.e., only few PUs are active, such characteristic of sparsity meets the assumption of compressed sensing (CS) [4], [5], [6], which is an evolutionary sampling theory that has received considerable attention recently.

Moreover, in wireless communications, the transmitted signals easily suffer from fading and noise interference. Fortunately, cooperative spectrum sensing (CSS) can be applied to all cooperative SUs that jointly sense the spectrums to better detect the status of spectrum usage [7], [8], [9]. Since the SUs in CSS share the same sparsity pattern for spectrum detection, joint sparsity model (JSM) proposed in [10] is suitable to model CSS. There exist some solutions to JSM. In this paper, we particularly focus on the solver, called Multiple Measurement Vectors (MMVs) [11], [12], which are composed of more

Corresponding author is C.-S. Lu (1cs@iis.sinica.edu.tw) than one measurement vector in the context of compressed sensing. However, CSS mainly cares about whether a channel is used or not, leading to the support detection problem. In other words, the number of active PUs in CSS corresponds to that of supports in CS.

\section{B. Related Work}

Sparse signal recovery or support detection from (far) fewer measurements can be achieved in two ways: matching pursuit (MP) and convex optimization (e.g., $\ell_{1}$ optimization). In the CSS literature, $\ell_{1}$ optimization is employed in [7], [8], incurring high complexity to delay and void the detection results. In this paper, we adopt orthogonal MP (OMP) as the solver due to its efficiency and computation simplicity. Although OMP is efficient, its recovery performance will be degraded due to the effect of fading and noise interference.

A crucial step in OMP is the stopping criterion that will affect its recovery performance. One popular stopping criterion for OMP is set to be the sparsity $K$ of a signal in that if $K$ supports are detected, then OMP stops its greedy iterations. Nevertheless, sparsity of a signal is usually unknown in advance. This is also the case in CSS. In order to deal with this problem, we study new stopping criteria, which are relatively unexplored in the literature, for OMP under the scenario of MMVs.

As for MMV solvers, SOMP (Simultaneous Orthogonal Matching Pursuit) [13] is considered to be a basic algorithm extended from OMP to MMVs. In [14], an advanced greedy algorithm, called Rank-Awareness Order Recursive Matching Pursuit (RA-ORMP), is proposed to deal with sparse signal recovery from MMVs, but without taking noisy measurements into account. In [9], Distributed OMP (DOMP) is presented to process measurement vectors in a distributed manner. Moreover, [15] proposes five thresholding-based algorithms; however, most of thresholds are designed to be related to sparsity $K$, which is, however, unknown in advance.

\section{Contributions}

Since noise interference will unavoidably make some supports un-detected, we mainly aim to detect those significant ones without necessarily recovering all. Specifically, we derive theoretical bounds as the stopping rules in a noisy MMVs environment to deal with noisy measurements. The derived bounds are simply constrained by the noise variance and measurement matrix's dimensionality, and are verified to be effective via simulations and comparisons. 
In addition to stopping criteria, we also introduce a novel subspace-based MMVs algorithm to improve support detection. In the literature, subspace-based algorithms [16], [17] have been applied to MMVs. Especially, [16] improves [17] to maintain detection quality with less measurement vectors when noise interference exists. In this paper, we modify [16] to adapt to our stopping criteria without needing to use the prior knowledge regarding sparsity. The derived stopping criteria are able to overcome the problem of unknown sparsity, and it can maintain good support detection rate. It also deserves to note that our stopping criteria can be combined to the existing greedy algorithms in CS in order to achieve robust sparse signal recovery or support detection.

We apply the proposed subspace-based MMVs algorithm to cooperative spectrum sensing in cognitive radio networks. Performance comparisons with the state-of-the-art MMVs methods are also provided.

\section{Multiple Measurement Vectors (MMVs)}

We consider an MMVs model to cope with the cooperative sparse spectrum sensing problem in cognitive radio networks.

\section{A. MMVs in Compressed Sensing}

MMV is one of the solutions to joint sparse model. Here, we consider the scenario that the multiple measurement vectors may be contaminated by noises.

Let the signal matrix $S \in \mathbb{R}^{N \times L}$ be composed of $L$ signals and be denoted as $S=\left[S_{1}, \ldots, S_{L}\right]$, where $S_{i} \in \mathbb{R}^{N}$ for $1 \leq i \leq L$. Given a specific dictionary $\Psi \in \mathbb{R}^{N \times N}$, we say that $S$ has a sparse representation in $\Psi$ and is denoted as $S=\Psi X$ if the corresponding transformed coefficient matrix is $X \in \mathbb{R}^{N \times L}$. Let $\Theta$ be the common support set of $X$, where $\Theta=\left\{i \mid X_{i,:} \neq 0, i \in 1, \ldots, N\right\}$ and $X_{i,:} \neq 0$ means that $i^{\text {th }}$ row are all with nonzero entries. The cardinality of $\Theta$ is defined as the sparsity $K$.

Let $\Phi \in \mathbb{R}^{M \times N}$ be the sensing matrix for MMVs, where $M<N$ and $\Phi$ is normalized columnwisely. Random projection of $X$ via $\Phi$ in a noisy environment can be expressed as:

$$
Y=\Phi S+\epsilon=\Phi \Psi X+\epsilon=A X+\epsilon,
$$

where $Y \in \mathbb{R}^{M \times L}$ denotes the measurement matrix, $\epsilon \in$ $\mathbb{R}^{M \times L}$, consisting of $\epsilon=\left[\epsilon_{1}, \ldots, \epsilon_{L}\right]$, denotes the noise, and $A=\Phi \Psi$ with each column vector being normalized. We say that $Y$ is composed of $L$ measurement vectors.

\section{B. MMVs in Cooperative Spectrum Sensing (CSS)}

In spectrum sensing, the target spectrum band is divided into $N$ individual sub-channels and $K$ active PUs on wideband spectrum are detected by $L$ SUs simultaneously. Moreover, PUs and SUs constitute a small-scale network, like femtocell network. Therefore, all SUs face the identical spectrum pattern, but observe signals of different power from PUs to generate a kind of MMVs from sensing the observed signals. Under the condition of MMVs, all SUs' measurement vectors are transmitted to a fusion center, where the original sparse information (status of spectrum usage) can be solved from a batch of measurements.
Formally, in a noisy spectrum sensing environment, $K$ PUs' transmission signals are aggregated to be a mixed analog signal $p_{j}$ and accompanied with additive white Gaussian noise $e_{j}$ in time domain with respect to the $j^{\text {th }} \mathrm{SU}(1 \leq j \leq L)$. Thus, the signal observed by SU $j$ is defined as $S_{j}=p_{j}+e_{j}$. Let the sensing matrix $\Phi$ be equipped in each SU. The measurement vector generated in SU $j$ is defined as $\delta_{j}=$ $\Phi S_{j}=\Phi p_{j}+\Phi e_{j}$, where $\delta_{j}$ is then transmitted to the fusion center. Let $Y_{j}$ be the data actually received at the fusion center and be defined as:

$$
Y_{j}=\delta_{j}+v_{j}=\Phi p_{j}+\Phi e_{j}+v_{j}=\Phi p_{j}+\epsilon_{j},
$$

where $v_{j}$ is noise incurred during the transmission path to the fusion center and $\epsilon_{j}$ is defined to be the final noise term.

Following [7], let the dictionary $\Psi$ be an $N \times N$ diagonal matrix, representing the status of PUs. If the diagonal entry is non-zero, it implies a sub-spectrum is occupied; otherwise, it is un-occupied. Therefore, we have:

$$
p_{j}=\Psi x_{j}
$$

Combining Eqs. (2) and (3), we have Eq. (1).

\section{ORACLE BOUND FOR STOPPING CRITERIA IN GREEDY MMVS ALGORITHMS}

We first introduce exact recovery condition and then describe the proposed stopping criteria for MMVs greedy algorithms.

\section{A. Exact Recovery Condition (ERC)}

We review exact recovery condition (ERC) [12][18] here because it is an important requirement to guarantee exact recovery to design oracle stopping criteria for MMVs greedy algorithms. Let $A(\Theta)$ be the set of columns, collected from $A$, that correspond to the elements in $\Theta$. Assume the first $w$ steps in a greedy algorithm achieve correct recovery with $W \subseteq \Theta$ being the set of detected supports. Similarly, let $A(W \bar{W})$ denote the set of columns collected from $A$ with respect to $W . A(W)$ spans a projection operator to be $P_{w}=$ $A(W)\left(A(W)^{T} A(W)\right)^{-1} A(W)^{T}=A(W) A(W)^{\dagger}$, where $\dagger$ means a pseudoinverse operator. Hence, in $w^{\text {th }}$ step, the residual $R_{w}$ can be derived as:

$$
R_{w}=\left(I-P_{w}\right) Y=\left(I-P_{w}\right) A X+\left(I-P_{w}\right) \epsilon=Q_{w}+H_{w},
$$

where $Q_{w}$ is the clear part and $H_{w}$ is the noisy part.

Furthermore, ERC derived as:

$$
\Pi=\max _{a \in A \backslash A(\Theta)}\left\|A(\Theta)^{\dagger} a\right\|_{1}<1,
$$

where $a$ denotes a column in $A$, is a sufficient condition to exactly recover sparse signals in both Single Measurement Vector (SMV) [18] and MMVs [12].

According to [18], ERC can be further derived for SMV to satisfy $\Pi \leq \frac{K \mu_{S}}{1-(K-1) \mu_{S}}<1$, where $K$ is sparsity and mutual incoherence regarding $A, \mu_{S}<\frac{1}{2 K-1}$ is a sufficient condition to have exact recovery in the case of noiseless SMV. Moreover, under the condition of MMVs [14], we can have:

$$
\Pi \leq \frac{K \mu_{M}}{1-(K-1) \mu_{M}}<1,
$$


where $\mu_{M}$ is the maximum incoherence regarding $A$ in MMVs. WLOG, mutual incoherence of $A$ is defined as $\mu=\max _{i \neq j}\left|a_{i}^{T} a_{j}\right|$. In the next subsection, we will describe the proposed stopping criteria in greedy MMVs algorithms.

\section{B. Stopping Criteria in Greedy MMVs Algorithms}

We let $G$ denote the set of remaining unsolved supports when a greedy algorithm proceeds to the $w^{t h}(<K)$ step. Then, we have $G=\Theta \backslash W$.

Based on Eq. (4), we first define three variables as follows that will be useful later. In $w^{\text {th }}$ step, let $B_{w}=$ $\max _{a \in A(G)}\left\{\left\|a^{T} Q_{w}\right\|_{2}\right\}$ be the maximum correlation between $Q_{w}$ and columns from $\Theta$, where $\|\cdot\|_{2}$ denotes $l_{2}$-norm. Let $E_{w}=\max _{a \in A}\left\{\left\|a^{T} H_{w}\right\|_{2}\right\}$ be the maximum correlation between columns of $A$ and $H_{w}$.

As in Eq. (1), let the corresponding coefficient matrix of $A(G)$ be denoted as $X(G)$. In order to realize the lower bound of $\|X(G)\|_{F}$, where $\|\cdot\|_{F}$ denotes Frobenius norm, that allows a greedy algorithm to have unique recovery, we first derive the bound of $B_{w}$ as:

$$
B_{w} \geq \frac{1-u \mu_{M}}{\sqrt{K-w}}\|X(G)\|_{F} .
$$

Please see Appendix A for details.

Next, the sufficient condition for choosing a correct support is derived as:

$$
\|X(G)\|_{F} \geq \frac{2 E_{w} \sqrt{K-w}\left[1-(K-1) \mu_{M}\right]}{\left(1-u \mu_{M}\right)\left[1-(2 K-1) \mu_{M}\right]},
$$

which is obtained from Eq. (7) and Eq. (19) in Appendix B, which derives the relationship between $E_{w}$ and $B_{w}$.

Now, we consider how to derive reasonable stopping criteria for MMV greedy algorithms. Such stopping criteria will be related to the residual $R_{w}$, which is iteratively updated according to Eq. (4), and $\left\|R_{w}\right\|_{F}$ becomes smaller as $w$ becomes larger. When $\left\|R_{w}\right\|_{F}$ is smaller than a threshold, the greedy algorithm stops. According to the results of (C.1) and (C.2) in Appendix C, we have:

$$
\begin{aligned}
\left\|R_{w}\right\|_{F} & =\left\|\left(I-P_{w}\right) A X+\left(I-P_{w}\right) \epsilon\right\|_{F} \\
& \geq\left\|\left(I-P_{w}\right) A X\right\|_{F}-\left\|\left(I-P_{w}\right) \epsilon\right\|_{F} \\
& \geq\left\|\left(I-P_{w}\right) A(G) X(G)\right\|_{F}-b_{F},
\end{aligned}
$$

where $b_{F}$ is an upper bound of $\|\epsilon\|_{F}$. More details will be described in Sec. III-C.

Furthermore, by means of the result, $E_{w} \leq b_{F}$, derived in Appendix $\mathrm{C},\|X(G)\|_{F}$ in Eq. (8) has a much tighter bound derived as:

$$
\begin{aligned}
\|X(G)\|_{F} & \geq \frac{2 b_{F} \sqrt{K-w}\left[1-(K-1) \mu_{M}\right]}{\left(1-u \mu_{M}\right)\left[1-(2 K-1) \mu_{M}\right]} \\
& \geq \frac{2 b_{F}\left[1-(K-1) \mu_{M}\right]}{\left(1-u \mu_{M}\right)\left[1-(2 K-1) \mu_{M}\right]} .
\end{aligned}
$$

By taking the facts that $\left\|\left(I-P_{w}\right) A(G) X(G)\right\|_{F} \geq$ $\left\|A(G)^{T}\left(I-P_{w}\right) A(G) X(G)\right\|_{F}$ due to dimension reduction and Eq. (17) in Appendix A, we can derive:

$$
\left\|\left(I-P_{w}\right) A(G) X(G)\right\|_{F} \geq \lambda_{\min }\|X(G)\|_{F} .
$$

As a result, according to Eqs. (9), (10), and (11), we finally derive that the Frobenius norm of residual at $w^{t h}$ step $\left\|R_{w}\right\|_{F}$ has a more strict lower bound as:

$$
\begin{aligned}
\left\|R_{w}\right\|_{F} & \geq \lambda_{\min }\|X(G)\|_{F}-b_{F} \\
& \geq\left(1-u \mu_{M}\right) \frac{2 b_{F}\left[1-(K-1) \mu_{M}\right]}{\left(1-u \mu_{M}\right)\left[1-(2 K-1) \mu_{M}\right]}-b_{F} \\
& =2 b_{F} \frac{1-(K-1) \mu_{M}}{1-(2 K-1) \mu_{M}}-b_{F} .
\end{aligned}
$$

By considering $\mu_{M}<\frac{1}{2 K-1}<1$ derived from Eq. (6) and the fact $\frac{1-(K-1) \mu_{M}}{1-(2 K-1) \mu_{M}}>1$, Eq. (12) can be further simplified as:

$$
\left\|R_{w}\right\|_{F} \geq 2 b_{F}-b_{F}=b_{F} .
$$

Therefore, a greedy algorithm stops once Eq. (13) is not satisfied.

\section{Upper Bound of Frobenius Norm of Gaussian Noise}

Based on Eq. (13), we now derive how $b_{F}$ is related to noise, i.e., $\|\epsilon\|_{F}$. This means that our stopping criteris can deal with noisy measurements in CSS. As shown in Appendix C, $b_{F}$ is related to $\|\epsilon\|_{F}$. Based on the assumption that the noise $\epsilon$, described in Eq. (1), is Gaussian distribution with $N\left(0, \sigma^{2}\right)$, we extend the derivation regarding the bound of $l_{2}$-norm of a Gaussian noise vector in SMV [19] to MMVs and obtain (details are omitted here):

$$
\begin{aligned}
P\left\{\|\epsilon\|_{F}\right. & \left.\leq\left[L \sigma^{2}(M+2 \sqrt{M \ln M})\right]^{\frac{1}{2}}\right\} \\
& \leq 1-M^{-L} L^{-\frac{1}{2}},
\end{aligned}
$$

where the probability of norm constraint approaches one due to $M>>1$ and $L>1$. We denote the first type of $b_{F}$ as $b_{F, 1}=\left[L \sigma^{2}(M+2 \sqrt{M \ln M})\right]^{\frac{1}{2}}$. We can check that when $L=1$, Eq. (14) is degenerated to the result of SMV in [19].

On the other hand, by considering the fact that Gaussian random noise cannot be precisely predicted, we alternatively seek to calculate the expectation of $\|\epsilon\|_{F}$, denoted as $E\left[\|\epsilon\|_{F}\right]$. Since square-root is concave, we can further derive via Jensen's inequality as:

$$
\mathrm{E}\left[\|\epsilon\|_{F}\right] \leq \sigma \sqrt{M L} .
$$

We denote the second type of $b_{F}$ as $b_{F, 2}=\sigma \sqrt{M L}$.

The two bounds derived in Eq. (14) and Eq. (15) are only related to the noise variance $\sigma^{2}$ and dimensionality of measurement matrix $Y$. We will evaluate their performance in Sec. V.

\section{Recovery Guarantee due to Distance between $S U \& P U$ in CSS}

In CSS, each nonzero entry of $X$ is an SU's received power $P_{s}$ modified from a PU's power $P_{p}$, both of which can be modeled as [20]: $P_{s}=P_{p}|h| d^{-\frac{\alpha}{2}}$, where $d$ denotes the distance between a pair of SU and PU, $\alpha$ denotes propagation loss factor, and $|h|$ is channel fading gain. We study the relationship between $d$ and recovery guarantee in the following.

As mentioned in Sec. III-C, when $L=1$ in a SMV case, a Gaussian noise vector has an upper bound like $\sigma \sqrt{M+2 \sqrt{M \ln M}}$. Hence, under the case of MMVs, $j^{\text {th }}$ SU suffers from individual noise effect with an upper bound, $b_{j}=\sigma_{j} \sqrt{M+2 \sqrt{M \ln M}}, 1 \leq j \leq L$. To simplify notations 
below, we assume all measurement vectors suffer from the same noise effect, i.e., $\sigma_{j}=\sigma$, so we have $b=b_{j}$ and $b_{F}=\left(\sum_{j=1}^{L} b_{j}^{2}\right)^{\frac{1}{2}}$ according to Eq. (14).

Based on the above, the lower bound of $l_{2}$-norm of each column of $X(G)$ in Eq. (10) can be derived to be equal to or larger than $\frac{2 b\left[1-(K-1) \mu_{M}\right]}{\left(1-u \mu_{M}\right)\left[1-(2 K-1) \mu_{M}\right]}$. Thus, in order to ensure to correctly obtain one of $K-w$ unsolved supports at step $w$, each absolute nonzero entry of $X(G)$ is equal to or larger than $\frac{1}{\sqrt{K-w}}\left\{\frac{2 b\left[1-(K-1) \mu_{M}\right]}{\left(1-u \mu_{M}\right)\left[1-(2 K-1) \mu_{M}\right]}\right\}$, as opposed to Eq. (10).

Given the above derived results and stable channel with fixed $\alpha$ and $|h|$, we have $P_{p}|h| d^{-\frac{\alpha}{2}}>$ $\frac{1}{\sqrt{K-w}}\left\{\frac{2 b\left[1-(K-1) \mu_{M}\right]}{\left(1-u \mu_{M}\right)\left[1-(2 K-1) \mu_{M}\right]}\right\}$, which indicates that distance upper bound between PU and SU, derived as:

$$
d<\left\{\frac{\sqrt{K-w} P_{p}|h|\left(1-u \mu_{M}\right)\left[1-(2 K-1) \mu_{M}\right]}{2 b\left[1-(K-1) \mu_{M}\right]}\right\}^{\frac{2}{\alpha}},
$$

is a sufficient condition to guarantee correct recovery of PUs' spectrum pattern at step $w$.

\section{Practical Subspace MMVs Greedy (PS-MMVs-G) Algorithm}

The existing MMVs greedy algorithms rely on some prior knowledge in that [9], [14], [16] have to know sparsity $K$ and [14] and [16] need the relationship between $K$ and $L$ : [14] and [16] require $L \geq K$ and $L<K$, respectively. Here, we introduce a practical subspace MMVs greedy (PS-MMVs-G) algorithm without depending on the above prior knowledge. The oracle stopping criteria described in Sec. III-B are adopted in our algorithm to provide feasible termination for greedy methods under noisy environment.

Meanwhile, greedy support detection for either SMV or MMVs relies on the correlation between measurement $Y$ and column subset of $A$. For the purpose of precise recovery, augmented subspace is proposed in [16] to extend from $Y$ to merge both column subset of $A$ and $Y$ as a new basis, which is also the subspace of $\operatorname{Range}(A(\Theta))$ in noiseless cases, to enhance support detection. The detail will be described in Sec. IV-B.

Algorithm 1 depicts the proposed method PS-MMVs-G.

\section{A. Initial Support Detection}

Since subspace-based algorithms initially need to be given reliable supports as space basis, we utilize SOMP [13] to obtain the first support. Even if low SNR exists, the first recovered support from SOMP is correct with very high probability.

\section{B. Detection of Remaining Supports: Advanced Subspace Method}

In [16], SVD of $Y$ is performed to have $U_{1} \Sigma_{1} V_{1}^{T}$ and $Y$ is transferred to have the canonical form, $Y_{C}=Y V_{1}$, for support detection later. For support detection, the minimum correlation between $a_{j}(\in A)$ and the projection matrix $P_{A(\Theta)}^{\perp}$, where $P_{A(\Theta)}^{\perp}=I-A(\Theta) A(\Theta)^{\dagger}$, is conducted.

Nevertheless, $A(\Theta)$ is never known in advance. An alternative is to do SVD of the new basis $\left[A_{w} Y_{C}\right]$ at step $w$ to get $U \Sigma V^{T}$, where $A_{w}$ denotes the column set collected from $A$ at the first $w-1$ steps, Range $\left(\left[\begin{array}{ll}A_{w} & Y_{C}\end{array}\right]\right)=\operatorname{Range}(U)$, and Range $\left(\left[\begin{array}{ll}A_{w} & Y_{C}\end{array}\right]\right) \subset \operatorname{Range}(A(\Theta))$. It should be noted that, for the purpose of finding a subspace of $A(\Theta)$ in [16], Range $\left(\left[\begin{array}{ll}A_{w} & Y_{C}\end{array}\right]\right)$ is replaced with the first $K$ columns of $U$, say $U_{K}$, to become a new basis, i.e., Range $\left(U_{K}\right) \subset$ Range $(A(\Theta))$ due to the fact that $K$ column vectors of $U_{K}$ are orthonormal basis and $\operatorname{Rank}\left(U_{K}\right)=\operatorname{Rank}(A(\Theta))=K$. Thus, the projection matrix is now based on $U_{K}$ instead of $A(\Theta)$. The question, however, is how can $K$ be known in advance? We deal with this problem in the following.

In our method, at $w^{\text {th }}$ step $(w \geq 2)$, the first $w-1$ (independent of $K$ ) columns from $U$ is collected as the set $U_{w}$. Additionally, let $B=\operatorname{Range}\left(U_{w}\right)$. Accordingly, the projection matrix $P_{B}^{\perp}$ based on $B$ becomes $I-B B^{\dagger}$. The support at position $j$ is then determined from the minimum correlation between $a_{j}(j \notin W)$ and $P_{B}^{\perp}$. This implies that our stopping criteria can provide robust support detection to perform greedy CS algorithm without depending on any prior knowledge.

The simulation results also confirm that our method achieves remarkably lower probability of false alarm than [16].

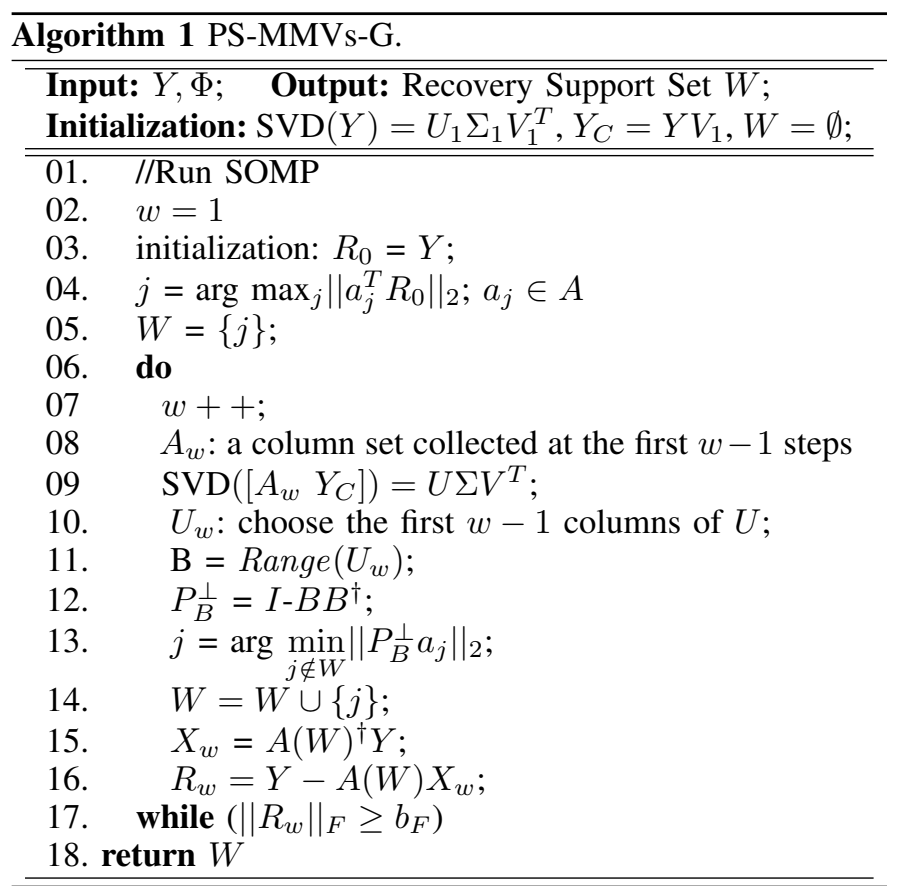

\section{Simulation Results}

\section{A. Simulation Setting}

Our simulations were conducted in terms of probability of detection (POD), probability of false alarm (PFA), and probability of miss detection (PMD) [7] to evaluate the performance of cooperative spectrum sensing in cognitive radio under noisy MMVs scenarios. The additive white Gaussian noise (AWGN) was distributed with $N\left(0, \sigma^{2}\right)$. As described in Sec. III-D, we set the channel fading gains with $|h|=1$ and propagation loss factor $\alpha=4$. PUs and SUs were assumed to be uniformly distributed in a small-scale network. A feasible CSS algorithm should be able to achieve high POD, and low PFA and PMD. Moreover, four state-of-the-art MMVs 
algorithms, i.e., DOMP [9], RA-ORMP [14], SOMP [13], and sequential CS-MUSIC [16], were adopted to compare with our algorithm in dealing with CSS.

Our proposed stopping rules, described in Sec. III-B, were used because, as we have mentioned before, the existing MMVs methods need some prior knowledge to stop that is impractical. Three stopping criteria were considered in our simulations and comparisons. The first criterion $R(1)$ is $\left\|R_{w}\right\|_{F}<b_{F, 1}=\left[L \sigma^{2}(M+2 \sqrt{M \ln M})\right]^{\frac{1}{2}}$, as defined in Eq. (14). The second criterion $R(2)$ is defined to be $\left\|R_{w}\right\|_{F}<b_{F, 2}=\sigma \sqrt{L M}$ based on Eq. (15). The third stopping rule $R(3)$ is conventional in that the ideal number of supports $K$ is known to exactly recover $K$ supports. Since DOMP assumes to know $K$ and may detect more than $K$ supports, and sequential CS-MUSIC does not have the concept of measurement residual, both did not adopt the above three criteria.

\section{B. Simulation under A fixed Set of Parameters}

We first observed simulation results under a fixed set of parameters, where the number $N$ of channels is 250 , number $M$ of measurements in a measurement vector is 50 , number $K$ of active PUs is 25 , number $L$ of cooperative SUs is 20 , and $\sigma^{2}=1 . L<K$ was set because CS-MUSIC [16] needs to satisfy this constraint.

Fig. 1 shows the results for PFA. We can find that, compared to Fig. 1(c) with stopping criterion $R(3)$ that needs to know $K$ in advance, both Fig. 1(a) with $R(1)$ and Fig. 1(b) with $R(2)$ are able to stop greedy support detection with significantly smaller PFA under various noise variances. Therefore, knowing the sparsity $K$ in advance not only is impractical but also exhibits higher PFA. We can find that DOMP attains the worst results.
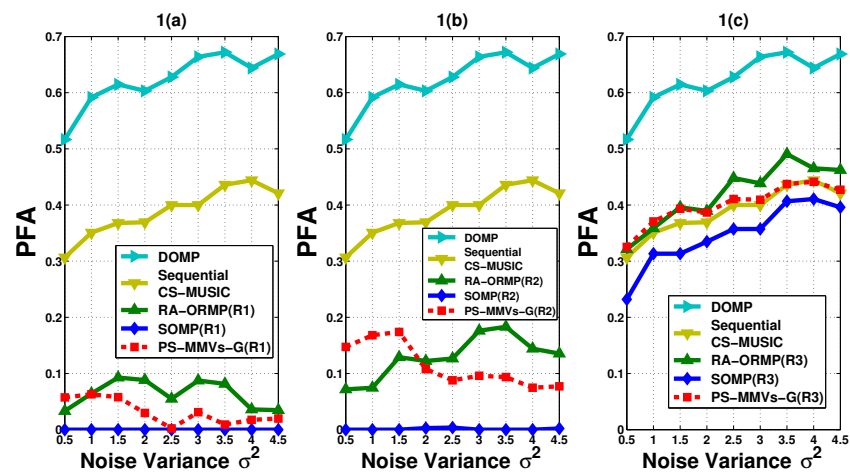

Fig. 1. PFA vs. noise variance under $N=250, M=50, K=25$, and $L=20$.

Based on the PFA results, we further observe results on POD and PMD, as shown in Fig. 2, without considering $R(3)$. In addition, DOMP and sequential CS-MUSIC were not considered for POD and PMD because they have incurred rather high PFA. With the fact that $b_{F, 1}>b_{F, 2}$, the criterion $R(1)$ may stop early to reach lower PFA and sacrifice fewer possible supports to be solved, leading to lower POD. In CSS applications, PFA means losing opportunity to access channels and PMD indicates the possibility of interference to PUs. A better strategy for SUs would rather take lower PMD instead of PFA, because protocol design in cognitive radio networks prohibits SUs from interfering active PUs. Under this situation, SUs aim to get lower PMD, which usually accompanies with higher POD.

We can observe from Fig. 2 that $R(2)$ achieves higher POD and lower PMD than $R(1)$ does. As for SOMP and DOMP, we can observe worse POD and PMD for both $R(1)$ and $R(2)$. As for RA-ORMP, its performance is inferior to PS-MMVs-G in $R(2)$. Especially, we can observe from Fig. 2(b) and Fig. 2(d) that our proposed algorithm PS-MMVs-G performs the best among the four algorithms.
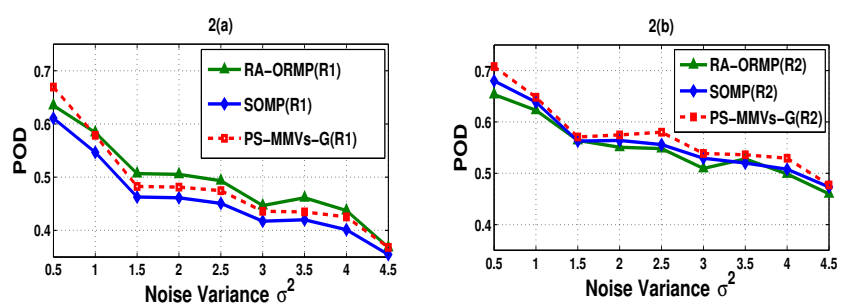

2(c)
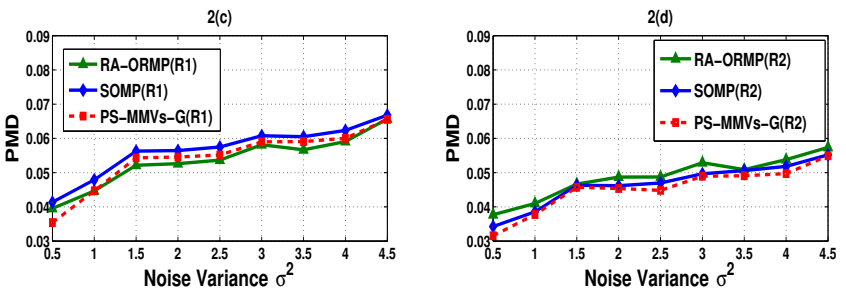

Fig. 2. POD and PMD vs. noise variance under $N=250, M=50$, $K=25$, and $L=25$.

\section{Simulations with Phase Transition}

Phase transitions of our method PS-MMVs-G with respect to PFA, POD, and PMD based on stopping criterion $R(2)$ are shown in Fig. 3. Both $K / M$ and $M / N$ range from 0.1 to 0.35 . Signal length $N$ is fixed and set to 250 .

Under fixed noise variance, the SNR of a sparse signal $X$ with $K$ nonzero entries is proportion to $K$. Therefore, a sparse signal with larger number $K$ of non-zero entries leads to higher POD and lower PFA. As for PMD [7], its denominator is equal to number $N$ of channels minus the number of detected supports, and dominates PMD. Thus, larger $K$ will lead to higher PMD.

The phase transition results indicate that, for a broad set of parameters, our method can obtain reasonable high POD and low PFA.

\section{Vi. Conclusions}

Joint sparsity model (JSM) is a good candidate to model the problem of CSS, as all SUs share the same sparsity pattern. Although MMVs is an efficient solver to JSM, it lacks robust stopping criterion to deal with noisy measurements. In this paper, we have presented theoretical bounds as the stopping criteria for MMVs and have combined them with an advanced subspace-based algorithm to practically conduct cooperative sensing for spectrum detection. Simulations have been provided to demonstrate the effectiveness of our algorithm to resist noise interference. 

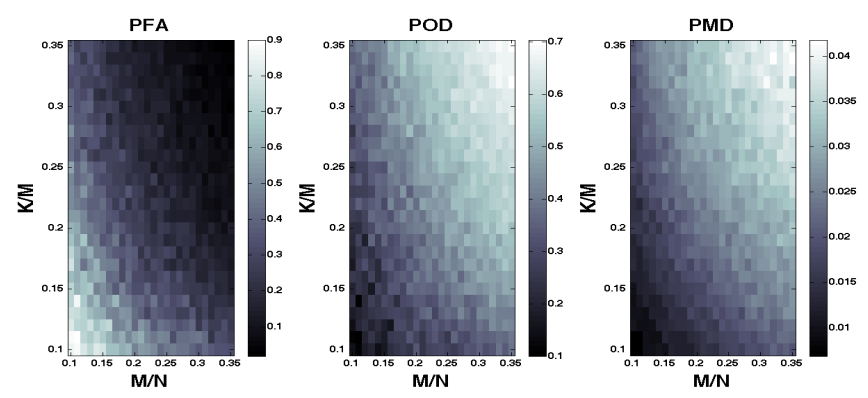

Fig. 3. Phase transition of PS-MMVs-G in $R(2)$ with $N=250$ and $\sigma^{2}=1$.

\section{ACKNOWLEDGMENT}

This work was supported by National Science Council, Taiwan, ROC, under grants NSC 102-2221-E-001-002-MY and NSC 102-2221-E-001-022-MY2.

\section{APPENDIX}

\section{APPENDIX A}

\section{LOWER BOUND OF $\|X(G)\|_{F}$}

As shown in [21], $\lambda_{\min } \geq 0$ is a sufficient condition for ERC, where $\lambda_{\min }$ is the minimum eigenvalue of $A(\Theta)^{T} A(\Theta)$. In [21], the authors show that $\lambda_{\min } \leq \lambda_{g}$, where $\lambda_{g}$ is the minimum eigenvalue of $A(G)^{T}\left(I-P_{w}\right) A(G) x_{g}$ and $x_{g}$ is one of columns from $X(G)$. Thus, the eigenfunction can be derived as:

$$
\begin{aligned}
\left\|A(G)^{T} q_{w}\right\|_{2} & =\left\|A(G)^{T}\left(I-P_{w}\right) A(G) x_{g}\right\|_{2} \\
& =\lambda_{g}\left\|x_{g}\right\|_{2} \geq \lambda_{\min }\left\|x_{g}\right\|_{2},
\end{aligned}
$$

where the column $q_{w} \in Q_{w}$.

Based on Eq.(17), we can derive the lower bound of $B_{w}$ in MMVs as:

$$
\begin{aligned}
B_{w} & =\max _{a \in A(G)}\left\{\left\|a^{T} Q_{w}\right\|_{2}\right\}=\max _{q_{w} \in Q_{w}}\left\|A(G)^{T} q_{w}\right\|_{2} \\
& \geq \frac{1}{\sqrt{K-w}}\left\|A(G)^{T} q_{w}\right\|_{F} \\
& \geq \frac{\lambda_{\text {min }}}{\sqrt{K-w}}\|X(G)\|_{F} \geq \frac{1-u \mu_{M}}{\sqrt{K-w}}\|X(G)\|_{F},
\end{aligned}
$$

where $0 \leq 1-u \mu_{M} \leq \lambda_{\min }$ holds, as in the SMV case in [21] and $u$ is a constant used to maintain $u \mu_{M} \leq 1$.

\section{APPENDIX B}

\section{RELATIONSHIP BETWEEN $E_{w}$ AND $B_{w}$}

Combining the results, $\frac{2 E_{w}}{1-\Pi}<B_{w}$ from [21] and $\Pi \leq$ $\frac{K \mu_{M}}{-(K-1) \mu_{M}}<1$ from Eq. (6), we can derive:

$$
2 E_{w} \frac{1-(K-1) \mu_{M}}{1-(2 K-1) \mu_{M}}<B_{w} .
$$

\section{APPENDIX C \\ RELATIONSHIP BETWEEN $E_{w}$ AND $b_{F}$}

Referring to the derivations for SMV in [21], we make extensions to MMVs and obtain the following conclusions: (C.1) $\left\|H_{w}\right\|_{F}=\left\|\left(I-P_{w}\right) \epsilon\right\|_{F} \leq\|\epsilon\|_{F} \leq b_{F}$, where $b_{F}$ will be derived in Sec. III-B, and (C.2) $E_{w}=$ $\left\|a^{T} H_{w}\right\|_{2}=\left(\sum_{i=1}^{L}\left(a^{T} H_{w}^{(i)}\right)^{2}\right)^{\frac{1}{2}}$, where $H_{w}^{(i)}$ is a column of $H_{w}(1 \leq i \leq L)$. Based on Cauchy-Schwarz inequality and $\|a\|_{2}=1$, we can further derive from (2) to attain $E_{w} \leq\left(\sum_{i=1}^{L}\|a\|_{2}^{2}\left\|H_{w}^{(i)}\right\|_{2}^{2}\right)^{\frac{1}{2}} \leq b_{F}$.

\section{REFERENCES}

[1] III Mitola, J. and Jr. Maguire, G.Q., "Cognitive radio: Making software radios more personal," Personal Communications, IEEE, vol. 6, no. 4, pp. 13-18, 1999.

[2] Federal Communication Commission, "Spectrum policy task force report," vol. 02, pp. 135, 2002.

[3] P. Paysarvi-Hoseini and N.C. Beaulieu, "Optimal wideband spectrum sensing framework for cognitive radio systems," Signal Processing, IEEE Transactions on, vol. 59, pp. 1170-1182, 2011.

[4] E. Candes, J. Romberg, and T. Tao, "Robust uncertainty principles: Exact signal reconstruction from highly incomplete frequency information," Information Theory, IEEE Transactions on, vol. 52, no. 2, pp. 489-509, 2006.

[5] D.L. Donoho, "Compressed sensing," Information Theory, IEEE Transactions on, vol. 52, no. 4, pp. 1289-1306, 2006.

[6] R. Baraniuk, “Compressive sensing," IEEE Signal Processing Magazine, vol. 24, no. 4, pp. 118-121, 2007.

[7] Jia Meng, Wotao Yin, Husheng Li, E. Hossain, and Zhu Han, "Collaborative spectrum sensing from sparse observations in cognitive radio networks," Selected Areas in Communications, IEEE Journal on, vol. 29, no. 2, pp. 327-337, 2011.

[8] Zeng Fanzi, Chen Li, and Zhi Tian, "Distributed compressive spectrum sensing in cooperative multihop cognitive networks," Selected Topics in Signal Processing, IEEE Journal of, vol. 5, no. 1, pp. 37-48, 2011.

[9] Thakshila Wimalajeewa and Pramod K. Varshney, "Cooperative sparsity pattern recovery in distributed networks via distributed-omp," in Acoustics, Speech and Signal Processing. IEEE International Conference on, 2013.

[10] D.Baron, M.F. Duarte, M.B. Wakin, S.Sarvotham, and R.G.Baraniuk, "Distributed compressive sensing," Tech. Rep., Rice University, Houston, 2005.

[11] S.F. Cotter, B.D. Rao, K. Engan, and K. Kreutz-Delgado, "Sparse solutions to linear inverse problems with multiple measurement vectors," Signal Processing, IEEE Transactions on, vol. 53, no. 7, pp. 2477-2488, 2005.

[12] Jie Chen and X. Huo, "Theoretical results on sparse representations of multiple-measurement vectors," Signal Processing, IEEE Transactions on, vol. 54, no. 12, pp. 4634-4643, 2006.

[13] Joel A. Tropp, Anna C. Gilbert, and Martin J. Strauss, "Algorithms for simultaneous sparse approximation: Part i: Greedy pursuit," Signal Process., vol. 86, no. 3, pp. 572-588, 2006.

[14] M.E. Davies and Y.C. Eldar, "Rank awareness in joint sparse recovery," Information Theory, IEEE Transactions on, vol. 58, no. 2, pp. 11351146, 2012.

[15] J.D. Blanchard, M. Cermak, D. Hanle, and Y. Jing, "Greedy algorithms for joint sparse recovery," Signal Processing, IEEE Transactions on, vol. 62, no. 7, pp. 1694-1704, 2014.

[16] Jong Min Kim, Ok Kyun Lee, and Jong-Chul Ye, "Improving noise robustness in subspace-based joint sparse recovery," Signal Processing, IEEE Transactions on, vol. 60, no. 11, pp. 5799-5809, Nov 2012.

[17] Jong Min Kim, Ok Kyun Lee, and Jong-Chul Ye, "Compressive music: Revisiting the link between compressive sensing and array signal processing," Information Theory, IEEE Transactions on, vol. 58, no. 1, pp. 278-301, 2012.

[18] J.A. Tropp, "Greed is good: Algorithmic results for sparse approximation," Information Theory, IEEE Transactions on, vol. 50, no. 10, pp. 2231-2242, 2004.

[19] T.T. Cai, Guangwu Xu, and Jun Zhang, "On recovery of sparse signals via 11 minimization," Information Theory, IEEE Transactions on, vol. 55, no. 7, pp. 3388-3397, 2009.

[20] T. S. Rappaport, Wireless Communications: Principles and Practice, 2nd edition, Prentice Hall, 2002.

[21] T.T. Cai and Lie Wang, "Orthogonal matching pursuit for sparse signal recovery with noise," Information Theory, IEEE Transactions on, vol. 57, no. 7, pp. 4680-4688, 2011. 